\title{
Diffusion in cellular microdomains: application to synapses
}

\author{
D. Holcman
}

This paper is dedicated to Angela.

\begin{abstract}
Modern technologies driven by biological questions such as single particle tracking or electron microscopy have revealed how molecular activity underlies cell physiology. Because direct measurements or live monitoring are not always possible, understanding cellular activity at the molecular level has greatly benefit from cell reconstruction which requires multiple data sources coming in particular from electrophysiological recordings. This reconstruction was made possible by the use of theoretical approaches using biophysical modeling, statistical physics and applied mathematics. Merging multiple data sources was applied successfully to better understand neuronal synapses, which are micro-contacts between neurons underlying neuronal communication in the brain. Because direct measurements at a single synapse are still possible, by integrating physiological information from various scales and using physical modeling and mathematical analysis of reaction-diffusion in micro-domains involving few molecules, several quantitative aspects of the synaptic function have recently emerged. We review here recent progress of diffusion in cellular microdomains, which is a fundamental phenomena underlying cellular biochemical activity and we focus specifically in the second part of this review on synaptic transmission and regulation.
\end{abstract}

\section{Introduction}

Over the past 40 years, various technological revolutions such as patch clamp recordings $[\mathbf{4 2}]$, confocal imaging, dye fluorescent molecules $[\mathbf{6 9}]$, single particle tracking have revealed the complexity of biological cellular processes: cells are complex and dynamically regulated factories where molecules are synthesized and used for a specific function. A single cell can be spatially and functionally separated in several microdomains (Figure 1) and this architecture serves multiple functions. An eukaryotic cell contains a nucleus where the genetic material is protected and regulated through gene activation. Defects in the nucleus architectural organization are responsible for several human diseases [41]. However nuclear organization, gene expression and maintenance of genome integrity remain poorly understood [67]. Many organelles play a fundamental role in protein cycle: before being transported by a yet unknown coding signaling to the correct location, proteins (see fig. 1) mature in Golgi apparatus while being synthesized in endoplasmic reticulum.

Key words and phrases. Diffusion in microdomains, small hole, Synapses, Laplace equation. This research was supported by an ERC-Starting Grant and a HFSP Research Grant. 


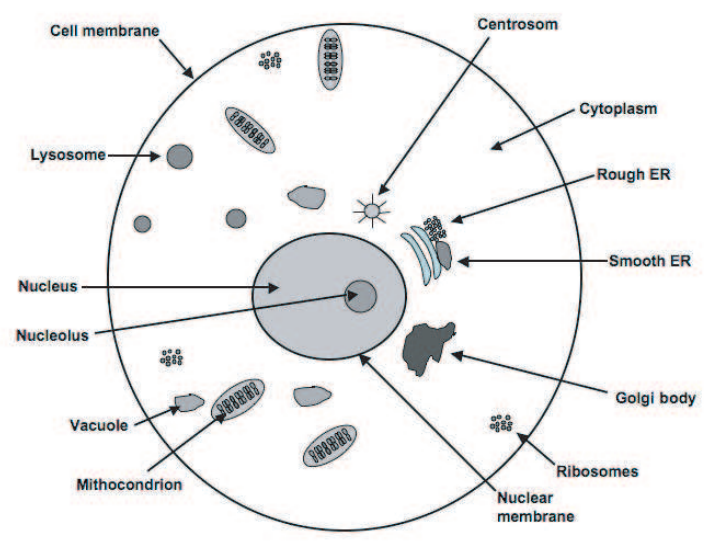

FiguRE 1. Schematic representation of an eukaryotic cell containing many organelles. Proteins have to travel from a compartment to another

Understanding the function of cellular microdomains and their interactions is still a challenging task of cellular biology that requires the participation of additional fields of science such as biophysics, statistical physics and applied mathematics. Many open questions remain unsolved in cellular biology that would require new possible framework. Examples are how memory is encoded in the brain, at which level and how it is retrieved? how cells build thresholds from the molecular level or how a cell can count events. We shall narrow our discussion to specific questions and consider now sub-cellular architecture. It plays a fundamental role in cell functions. For example, the rod photoreceptor outer-segment is a long cylinder, made of around 1000 coupled compartments, hosting the early chemical reactions of phototransduction. Amazingly a single microdomain can detect a unique photon $[\mathbf{4 7}]$. Degenerescence of this structure is associated with blindness and altered night vision. It is known that Viagra interferes with the phototransduction molecular cascade and thus locally disrupts night vision. In general, the analysis of the photon response has benefitted from the constant cross-talk between electrophysiology recordings, electron-microscopy image reconstruction and mathematical modeling (see figure 2). To illustrate the fundamental role of physical modeling and mathematical analysis in revealing both qualitatively and quantitatively the function of cell microdomains, we shall focus here on the neuronal synapse, involved in direct neuronal communication between two neighboring neurons. The role of neuronal synapses has originally been recognized more than a century ago by the early systematic analysis of Ramon y Cajal (fig 3), and since then, has emerged as a dominant field of neuroscience. The reason for this success is the central role of synaptic modifications in learning and memory and synaptic degeneration or defects, occurring in many neurological disorders. In addition, because direct measurements at a synaptic level are not possible, indirect analysis have been used to identify key principles of synaptic transmission: for example electron microcopy images [7] (fig 3) have shown the complexity of synapse hyper structure and local organelle organization. Patch-clamp recordings have allowed to record 

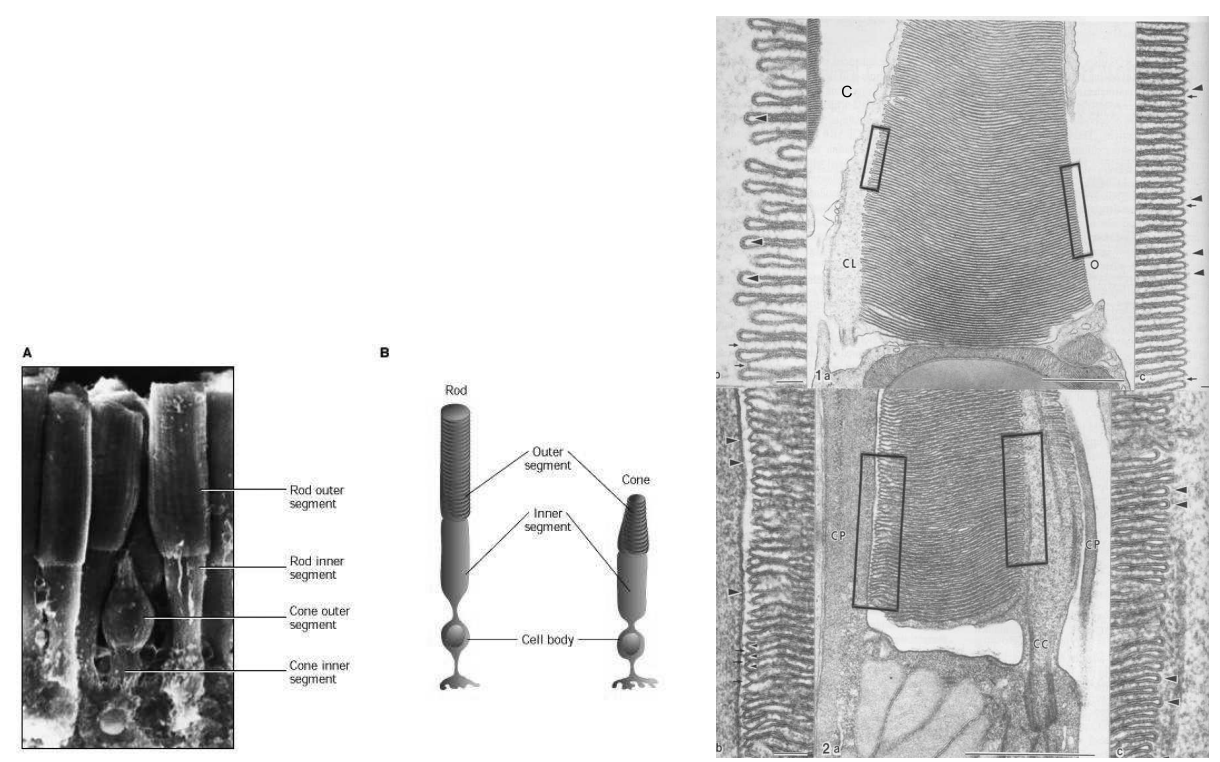

Figure 2. Photoreceptors are composed of an inner and outersegment, which are specific microdomains. A: example of densely packed rod and cone photoreceptors.B: schematic representation of the photoreceptor architectures. C: Electron Microscopy picture of a cone outersegment. The small boxes magnified on the sides show the complex architecture of the microdomains.

the electrical activity of single channels under various conditions $[42],[43]$. Neuronal recordings showed how local depolarization can lead to an action potential (fig 4). Finally, single receptor trafficking and related analysis have confirmed that receptors at synapses are not fixed, but are moving, affecting continuously synaptic transmission $[\mathbf{8}, \mathbf{1 0}, \mathbf{6 8}, \mathbf{2 5}]$. Because there are no systematic ways to integrate all of these data to reconstruct synaptic activity, over the past ten years, several modeling approaches were used to reconstruct the synaptic function $[\mathbf{2 9}, \mathbf{3}, \mathbf{1 7}]$. Synaptic dynamics has been explored using Brownian simulations or by solving partial differential reaction-diffusion chemical equations. Because numerical simulations can only provide a partial integrated view of the synaptic response and can be assimilated to an ideal experiment, the identification of physical rules and the formulation of mathematical principles remain key steps to quantify the synaptic activity. Identifying principles requires in particular the derivation of analytical formula from biophysical considerations. I believe that predicting and quantifying synaptic activity could be achieved by deriving mathematical formula, which synthesizes fundamental laws. The first part of this review is dedicated to some general principles of diffusion in cellular microdomains and in the second part, several new results related to synaptic transmission are presented.

\section{Diffusion principle in cellular microdomains}

The function of cellular microdomains depends on functional proteins that should reach the location where they are needed. For example, at functional 


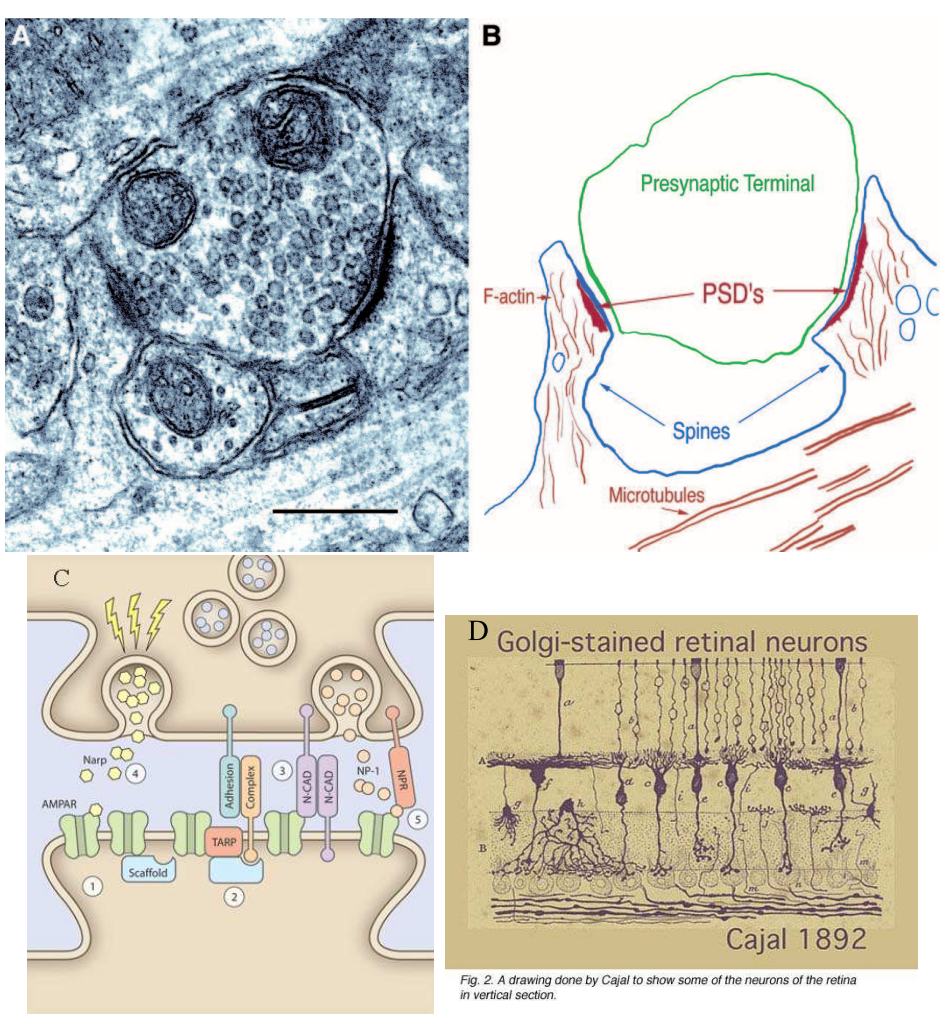

Figure 3. Neuronal synapse. Fig 3a-b represent a synapse with the postsynaptic density containing glutamatergic receptors (ref.[34]).Fig 3c is a schematic representation of the complex molecular organization of the synapse: neurotransmitters are released from fused vesicles (pre-synaptic part) and upon activation of gated receptors (represented in green), an ionic flow is generated in the postsynaptic terminal that leads to cellular depolarization. In between the pre and post-synaptic terminal, adhesion molecules maintain the synaptic structure. Fig $3 \mathrm{~d}$ is the legendary drawing of Cajal showing the retinal neural organization containing many synapses.

synapses, receptors are found in a microdomain called the post-synaptic density (see fig. 3). In addition, the function of several microdomains depends on chemical reactions involving few molecules that have to find their targets in a complex and crowded environment. To estimate the rate of chemical reactions in confined domains, we have studied the dynamics of a Brownian particle (molecule, protein) confined to a compartment by a reflecting boundary, except for a small window through where it can escape $[\mathbf{2 6}, \mathbf{5 6}, \mathbf{5 7}, \mathbf{5 8}]$. This problem, known as the narrow escape problem in diffusion theory also called the narrow escape time (NET), goes back to Lord Rayleigh [49]. The small hole often represents a small target on a cellular membrane, such as a protein channel, which is a target for ions [21], a 


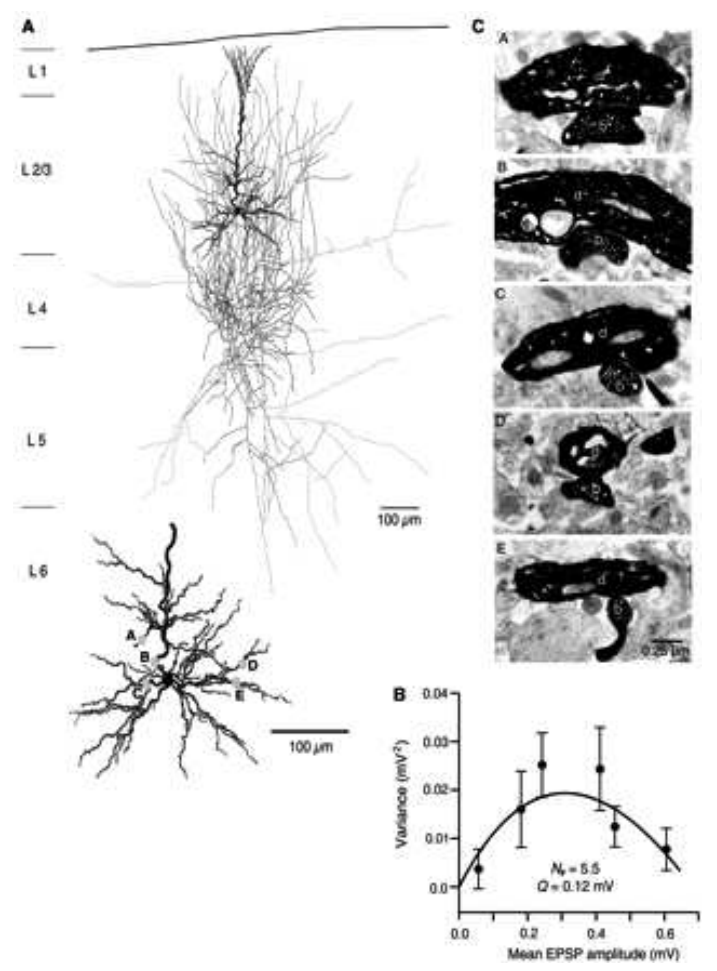

Figure 4. Two connected neurons have multiple synaptic boutons (4 to 6 ): the number of functional release sites and the number of anatomically identified synaptic contacts are equal at connections between spiny stellate and pyramidal cells in rat barrel cortex (Silver R, Science 302 (5652):1981-4.2003)

receptor for neurotransmitter molecules in a neuronal synapse [39, 43], a narrow neck in the neuronal spine [36], which is a target for calcium ions, and so on.

The physiological role of the small hole is often to regulate flux, which carries a physiological signal. For example, the NMDA and AMPA channels in the postsynaptic membrane in the neuronal cleft are small targets for diffusing glutamate molecules released from a vesicle at the presynaptic membrane. The problem here is to find the fraction of the released molecules that reach the channels before being irreversibly absorbed by the surrounding glial cells. The position and the number of AMPA receptors can regulate synaptic transmission [66].

The narrow escape problem is to calculate the mean sojourn time before it escapes. This time diverges as the window shrinks, thus rendering the calculation of the mean escape time a singular perturbation problem. Once formulated in terms of boundary value problems for partial differential equations, their singular perturbation analysis yields explicit asymptotic expressions for the mean escape time, depending on the diffusion coefficient, the ambient potential, dimensions, local and global geometrical properties of the domain and its boundary. The narrow escape problem is connected to that of calculating the principal eigenvalue of the mixed Dirichlet-Neumann problem for the Laplace equation in a bounded domain, 
whose Dirichlet boundary is only a small patch on the otherwise Neumann boundary $[\mathbf{1 6}, \mathbf{2 8}]$. Specifically, the principal eigenvalue is asymptotically the reciprocal of the narrow escape time in the limit of shrinking patch. The recent history of the problem begins with the works of Fabrikant, Keller, Ward, Henshaw, Van De Velde, and Kolokolnikov $[\mathbf{1 6 , 7 0 , 7 1 , 3 5}$ on the principal eigenvalue and is based on boundary layer theory and matching asymptotics, in which the boundary layer equation is the classical electrified disk problem, solved explicitly by Weber in 1873 $[\mathbf{3 0}]$. We have obtained various asymptotic formulas for the mean sojourn time in several cases $[\mathbf{2 6}, \mathbf{5 6}, \mathbf{6 6}]$, including regular domains in two and three dimensions and some singular domains in two dimensions.

Physical formulation: Random motion is overdamped medium. We shall now recall the biophysical context and the derivation of the Mean First Passage Time equations. We assume that a particle (e.g., protein, receptor, molecule) in a biological medium is Brownian in a field of force, so its motion can be described by the overdamped Langevin equation (known as the Smoluchowski limit), equation

$$
\dot{\boldsymbol{x}}-\frac{1}{\gamma} \boldsymbol{F}(\boldsymbol{x})=\sqrt{2 D} \dot{\boldsymbol{w}},
$$

where

$$
D=\frac{k_{B} T}{m \gamma}
$$

$\gamma$ is the friction coefficient per unit of mass, $\boldsymbol{F}(\boldsymbol{x})$ the force per unit of mass, $T$ is absolute temperature, $\mathrm{m}$ is the mass of the molecule, $k_{B}$ is Boltzmann's constant, and $\dot{\boldsymbol{w}}$ is a vector of independent $\delta$-correlated Gaussian white noise, which represents the effect of the thermal motion. The derivation of the Smoluchowski equation (1.1) is given in [64] for the three-dimensional motion of a molecule in solution, where Einstein's formula (1.2) can be applied. In two dimensions, such as for diffusion of a transmembrane protein in a cell surface, the diffusion coefficient was estimated by the Saffman-Delbrück formula [54]

$$
D=\frac{k_{B} T}{4 \pi \mu h}\left(\log \frac{\mu h}{\mu^{\prime} a}-\gamma_{E}\right)
$$

where $\mu$ is the viscosity of the solution, $\mu^{\prime}$ is the viscosity of the membrane, $h$ the length of the cylinder, $a$ is its radius, and $\gamma_{E}$ is Euler's constant $0.5772 \ldots$.

We now recall the estimation for the mean sojourn time of a Brownian particle in a bounded domain $\Omega$, before it escapes through a small absorbing window $\partial \Omega_{a}$ in its boundary $\partial \Omega$. The remaining part of the boundary $\partial \Omega_{r}=\partial \Omega-\partial \Omega_{a}$ is assumed reflecting for the particle. The reflection may also represent a high potential barrier on the boundary, or an actual physical impenetrable obstacle. The opening may represent a narrow corridor in the barrier, or a defect in the physical obstacle (see figure 5). The biological interpretation of the mean sojourn time is discussed below.

Asymptotic computation of the Narrow Escape Time. The escape time can be estimated asymptotically $[\mathbf{6 3}]$ in the limit

$$
\varepsilon=\frac{\left|\partial \Omega_{a}\right|}{|\partial \Omega|} \ll 1 .
$$




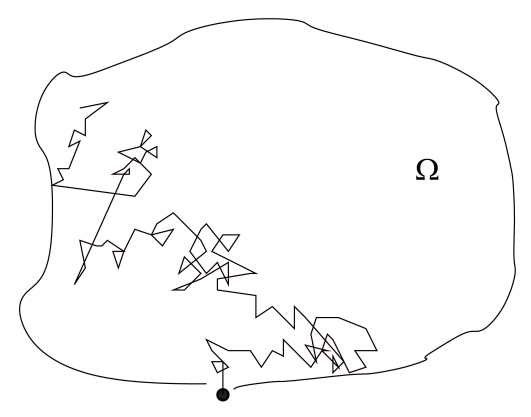

FiguRE 5. The escape of a Brownian trajectory through a small window.

The probability density function (pdf) $p_{\varepsilon}(\boldsymbol{x}, t)$ of the trajectories of (1.1) is the probability per unit volume (area) of finding the Brownian particle at the point $\boldsymbol{x}$ at time $t$ prior to its escape. The pdf satisfies the Fokker-Planck equation

$$
\frac{\partial p_{\varepsilon}(\boldsymbol{x}, t)}{\partial t}=D \Delta p_{\varepsilon}(\boldsymbol{x}, t)-\frac{1}{\gamma} \nabla \cdot\left[p_{\varepsilon}(\boldsymbol{x}, t) \boldsymbol{F}(\boldsymbol{x})\right] \triangleq L p_{\varepsilon}(\boldsymbol{x}, t),
$$

with the initial condition

$$
p_{\varepsilon}(\boldsymbol{x}, 0)=\rho_{0}(\boldsymbol{x}),
$$

and the mixed Dirichlet-Neumann boundary conditions $t>0$

$$
\begin{aligned}
p_{\varepsilon}(\boldsymbol{x}, t) & =0, \text { for } \boldsymbol{x} \in \partial \Omega_{a}, \\
D \frac{\partial p_{\varepsilon}(\boldsymbol{x}, t)}{\partial n}-\frac{p_{\varepsilon}(\boldsymbol{x}, t)}{\gamma} \boldsymbol{F}(\boldsymbol{x}) \cdot \boldsymbol{n}(\boldsymbol{x}) & =0, \text { for } \boldsymbol{x} \in \partial \Omega_{r},
\end{aligned}
$$

where $\rho_{0}(\boldsymbol{x})$ is the initial pdf $\left(\mathrm{e} . \mathrm{g}, \rho_{0}(\boldsymbol{x})=1 /|\Omega|\right.$ for a uniform distribution or $\rho_{0}(\boldsymbol{x})=\delta(\boldsymbol{x}-\boldsymbol{y})$, when the molecule is initially located at position $\left.\boldsymbol{y}\right)$. In the later case, the function

$$
u_{\varepsilon}(\boldsymbol{y})=\int_{\Omega} d \boldsymbol{x} \int_{0}^{\infty} p_{\varepsilon}(\boldsymbol{x}, t \mid \boldsymbol{y}) d t,
$$

where $p_{\varepsilon}(\boldsymbol{x}, t \mid \boldsymbol{y})$ is the pdf conditioned on the initial position, represents the mean conditional sojourn time. It is the solution of the boundary value problem [64]

$$
\begin{array}{r}
L^{*} u_{\varepsilon}(\boldsymbol{y}) \triangleq D \Delta u_{\varepsilon}(\boldsymbol{y})+\frac{1}{\gamma} \boldsymbol{F}(\boldsymbol{y}) \cdot \nabla u_{\varepsilon}(\boldsymbol{y})=-1, \text { for } \boldsymbol{y} \in \Omega \\
u_{\varepsilon}(\boldsymbol{y})=0, \text { for } \boldsymbol{y} \in \partial \Omega_{a} \\
\frac{\partial u_{\varepsilon}(\boldsymbol{y})}{\partial n}=0, \text { for } \boldsymbol{y} \in \partial \Omega_{r} .
\end{array}
$$

Equation (1.12) is the adjoint boundary condition to (1.8). The survival probability is

$$
S_{\varepsilon}(t)=\int_{\Omega} p_{\varepsilon}(\boldsymbol{x}, t) d \boldsymbol{x},
$$

where

$$
p_{\varepsilon}(\boldsymbol{x}, t)=\int_{\Omega} p_{\varepsilon}(\boldsymbol{x}, t \mid \boldsymbol{y}) \rho_{0}(\boldsymbol{y}) d \boldsymbol{y}
$$


The density $p_{\varepsilon}(\boldsymbol{x}, t \mid \boldsymbol{y})$ can be computed by expanding in eigenfunctions

$$
p_{\varepsilon}(\boldsymbol{x}, t \mid \boldsymbol{y})=\sum_{i=0}^{\infty} a_{i}(\varepsilon) \psi_{i, \varepsilon}(\boldsymbol{x}) \psi_{i, \varepsilon}(\boldsymbol{y}) e^{-\lambda_{i}(\varepsilon) t},
$$

where $\lambda_{i}(\varepsilon)$ (resp. $\psi_{i, \varepsilon}$ ) are the eigenvalues (resp. eigenfunctions) of the FokkerPlanck operator $L$ in $(1.5),(1.7),(1.8)$, and the coefficients $a_{i}(\varepsilon)$ depend on the initial function $\rho_{0}(\boldsymbol{y})$. The mean sojourn time can be expressed by integrating expansion 1.15 [64],

$$
u_{\varepsilon}(\boldsymbol{y})=\sum_{i=0}^{\infty} \frac{a_{i}(\varepsilon)}{\lambda_{i}} \psi_{i, \varepsilon}(\boldsymbol{y}) .
$$

When $\varepsilon>0$, the eigenvalues are strictly positive so the steady state is

$$
\lim _{t \rightarrow \infty} p_{\varepsilon}(\boldsymbol{x}, t \mid \boldsymbol{y})=0
$$

(the Brownian particle escapes in finite time with probability 1) and the mean sojourn time is asymptotically $1 / \lambda_{0}(\varepsilon)$, when $\varepsilon<<1$. Thus $\lambda_{0}(\varepsilon) \rightarrow 0$ as $\varepsilon \rightarrow 0$. For free Brownian motion (with $\boldsymbol{F}(\boldsymbol{x})=\mathbf{0}$ ), $\lambda_{1}(\varepsilon)=O(1)$ in this limit. Approximating the pdf, solution of equation 1.8 by

$$
p_{\varepsilon}(\boldsymbol{x}, t) \approx a_{0}(\varepsilon) \psi_{0}(\boldsymbol{x}) \psi_{0}(\boldsymbol{y}) e^{-\lambda_{0}(\varepsilon) t} \quad \text { for } \quad t \gg \frac{1}{\lambda_{1}(\varepsilon)},
$$

shows that under the small hole approximation, the survival probability is exponentially distributed,

$$
S_{\varepsilon}(t) \approx e^{-\lambda_{0}(\varepsilon) t} \quad \text { for } \quad t \gg \frac{1}{\lambda_{1}(\varepsilon)},
$$

because

$$
a_{0}(\varepsilon)=\int_{\Omega} \rho_{0}(\boldsymbol{x}) d \boldsymbol{x}=1, \quad \int_{\Omega} \psi_{0}(\boldsymbol{x}) d \boldsymbol{x}=1,
$$

due to normalization. If the medium contains initially $N_{0}$ independent particles, the population $N(t)$ decays exponentially with rate $\lambda_{0}(\varepsilon)$,

$$
N(t)=N_{0} S_{\varepsilon}(t) \sim N_{0} e^{-\lambda_{0}(\varepsilon) t} \quad \text { for } \quad \varepsilon \ll 1, \quad t>\frac{1}{\lambda_{1}(\varepsilon)} .
$$

The asymptotic solution of equations (1.10)-(1.12) depends on the dimension (2 or 3 ) and the local geometry near the small opening $[\mathbf{2 6}, \mathbf{5 6}, \mathbf{5 7}, \mathbf{5 8}]$. When the boundary of the domain is regular, the escape time $u_{\varepsilon}(\boldsymbol{y})$ is given for $\varepsilon \ll 1(\varepsilon$ is the angle of the absorbing boundary) by

$$
u_{\varepsilon}(\boldsymbol{y})=\left\{\begin{array}{cc}
\frac{A}{\pi D} \ln \frac{1}{\varepsilon}+O(1) & \text { for } \quad n=2 \\
\frac{V}{4 a D}[1+o(1)] & \text { for } \quad n=3,
\end{array}\right.
$$

where $a$ represents the small radius of a geodesic disk located on the surface of the domain $\Omega$ and depending on the dimension, $A$ (resp $V$ ) is the surface (resp. volume) of the domain $\Omega$. The function $u_{\varepsilon}(\boldsymbol{y})$ does not depend on the initial position $\boldsymbol{y}$, except for a small boundary layer near $\partial \Omega_{a}$, due to the asymptotic form found in $[26,56,57,58]$. 
In dimension 2, the first order term matters, because, for example, if $\varepsilon \approx 10^{-1}$, then $\ln \frac{1}{\varepsilon} \approx 2.3$ so the second term in the expansion (1.21) is comparable to the leading term. The second term can be found when $\Omega$ is a circular disk of radius $R$ and the particle starts at the center $[\mathbf{5 7}, \mathbf{5 8}]$, as

$$
E[\tau \mid \boldsymbol{x}(0)=\mathbf{0}]=\frac{R^{2}}{D}\left[\log \frac{1}{\varepsilon}+\log 2+\frac{1}{4}+O(\varepsilon)\right] .
$$

The escape time, averaged with respect to a uniform initial distribution in the disk, is given by

$$
E \tau=\frac{R^{2}}{D}\left[\log \frac{1}{\varepsilon}+\log 2+\frac{1}{8}+O(\varepsilon)\right] .
$$

The geometry of the small opening can affect the escape time: if the absorbing window is located at a corner of angle $\alpha$, then

$$
E \tau=\frac{|\Omega|}{\alpha D}\left[\log \frac{1}{\varepsilon}+O(1)\right] .
$$

More surprising, near a cusp in a two dimensional domain, the escape time $E \tau$ grows algebraically, rather than logarithmically: in the domain bounded between two tangent circles, the escape time is

$$
E \tau=\frac{|\Omega|}{(d-1) D}\left(\frac{1}{\varepsilon}+O(1)\right)
$$

where $d>1$ is the ratio of the radii. The mean time to escape near a cusp or any generic singularities in dimension 3 or higher is an open question that would have many consequences on estimating the search time. Finally, when the domain is an annulus, the escape time to a small opening located on the inner circle involve a second parameter which is $\beta=\frac{R_{1}}{R_{2}}<1$, the ratio of the inner to the outer radii, the escape time, averaged with respect to a uniform initial distribution, is

$$
\begin{aligned}
E \tau= & \frac{\left(R_{2}^{2}-R_{1}^{2}\right)}{D}\left[\log \frac{1}{\varepsilon}+\log 2+2 \beta^{2}\right]+\frac{1}{2} \frac{R_{2}^{2}}{1-\beta^{2}} \log \frac{1}{\beta} \\
& -\frac{1}{4} R_{2}^{2}+O\left(\varepsilon, \beta^{4}\right) R_{2}^{2} .
\end{aligned}
$$

In general, the link between the mean first passage time $E \tau$ and the eigenvalues is given by

$$
E \tau=\sum_{i=1}^{\infty} \frac{1}{\lambda_{i}} \approx \frac{1}{\lambda_{1}}+O(1) .
$$

This formula is valid in the small target limit: the first eigenvalue contributes significantly to the mean first passage time, while the sum of the rest is of order 1.

Refinement of the Narrow Escape Time: the role of the mean boundary curvature. To determine the refine structure of the narrow escape time (NET) in dimension 3, we recall that because the target is on the boundary, there is an additional singularity that we shall explain now: the Neumann function is solution of the Poisson equation and the Green-Neumann function for the mixed problem 
(with Dirichlet and Neumann boundary conditions) in a general bounded domain $\Omega$, whose boundary $\partial \Omega$ is sufficiently regular satisfies

$$
\begin{aligned}
\Delta_{\boldsymbol{x}} N(\boldsymbol{x}, \boldsymbol{y}) & =-\delta(\boldsymbol{x}-\boldsymbol{y})+\frac{1}{|\Omega|}, \quad \text { for } \quad \boldsymbol{x}, \boldsymbol{y} \in \Omega \\
\frac{\partial N(\boldsymbol{x}, \boldsymbol{y})}{\partial \nu_{\boldsymbol{x}}} & =0, \quad \text { for } \quad \boldsymbol{x} \in \partial \Omega, \boldsymbol{y} \in \Omega
\end{aligned}
$$

Using the method of $[\mathbf{4 6 , 5 2 ] : ~ f o r ~} \boldsymbol{z} \in \partial \Omega, \boldsymbol{y} \in \Omega \cup \partial \Omega$, the structure of the Neumann function (in dimensionless variables) is given by $[\mathbf{6 0}]$

$$
N(\boldsymbol{y}, \boldsymbol{z})=\frac{1}{2 \pi|\boldsymbol{y}-\boldsymbol{z}|}-\frac{1}{8 \pi}[L(\boldsymbol{z})+N(\boldsymbol{z})] \ln |\boldsymbol{y}-\boldsymbol{z}|+v_{S}(\boldsymbol{y}, \boldsymbol{z})
$$

where $L(\boldsymbol{z})$ and $N(\boldsymbol{z})$ are the principal curvatures of $\partial \Omega$ at $\boldsymbol{z}$ and $v_{S}(\boldsymbol{y}, \boldsymbol{z})$ is a bounded function of $\boldsymbol{x}, \boldsymbol{y}$ in $\Omega$. If $\Omega$ is a ball of radius $R$, the above mentioned result of Kellog $[\mathbf{3 3}]$ is recovered, because $L(\boldsymbol{z})=N(\boldsymbol{z})=\frac{1}{R}$. The NET through a circular disk of (dimensionless) radius $a$, centered at $\mathbf{0}$ on the boundary, is

$$
E \tau=\frac{|\Omega|}{4 a D\left[1+\frac{L(\mathbf{0})+N(\mathbf{0})}{2 \pi} a \log a+o(a \log a)\right]}
$$

where $D$ is the diffusion coefficient. It would be interesting to push forward this expansion from one term, to analyze the log-term for a general domain. When $\Omega$ is a ball of radius $R$, we get

$$
E \tau=\frac{|\Omega|}{4 a D}\left[1+\frac{a}{\pi R} \log \frac{R}{a}+o\left(\frac{a}{R} \log \frac{R}{a}\right)\right] .
$$

The result (1.32) corrects that given in [56], where $\pi$ is missing from the prelogarithmic factor.

This analysis can be used to estimate for a leaky conductor (one small hole and a large one) [60] the leakage flux through a circular hole of small (dimensionless) radius $\varepsilon$, centered at $\mathbf{0}$, it is

$$
J_{\varepsilon}=4 \varepsilon D u_{0}(\mathbf{0})(1+O(\varepsilon \log \varepsilon))
$$

where $u_{0}(\mathbf{0})$ is the solution of the unperturbed problem 1.10 (without the leak) at the center where the hole is located.

The case of multiple targets. The case of several targets is not as simple as thought and the associated mixed problem for a Dirichlet boundary $\partial \Omega_{a}$ consists of a collection of small windows embedded in an otherwise Neumann boundary $\partial \Omega_{r}$ of a regular bounded domain $\Omega$. Contrary to the case of a single window, the MFPT of a Brownian particle to many targets $\partial \Omega_{a}$ contains additional information about their relative position. In particular, when the small Dirichlet windows form a cluster, the MFPT to one of them is influenced by the others, which is not the case for well separated windows $[\mathbf{2 8 , 2 7}$. 


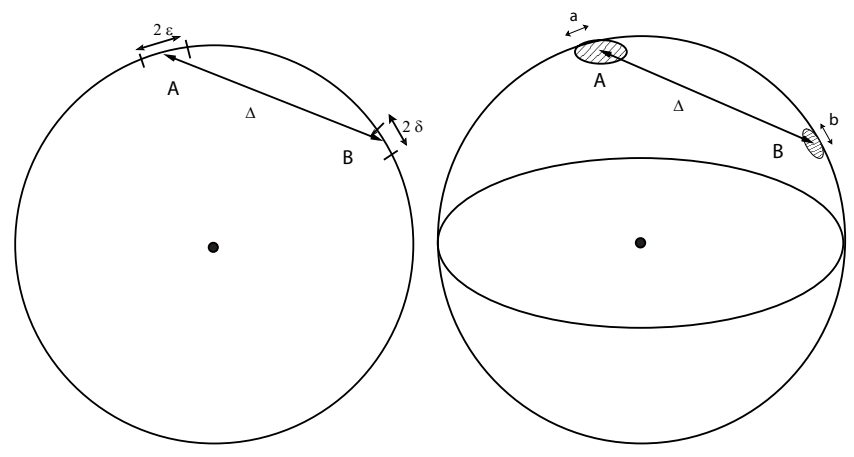

FiguRE 6. Schematic representation of a disk and a sphere with two holes on the boundary. In the plane, the arclengths of the holes are $2 \delta$ and $2 \varepsilon$, respectively, separated Euclidean distance $\Delta$, while in $3 \mathrm{D}$, the radii of the holes are, respectively, $a$ and $b$.

Explicit NET for two windows. For dimension $d=2$ and a regular domain with two Dirichlet arcs of lengths $2 \varepsilon$ and $2 \delta$ (normalized by the perimeter $|\partial \Omega|$ ) and separated by the Euclidean distance $\Delta=\varepsilon+\Delta^{\prime}+\delta$ between their centers, and in dimension $d=3$ for two Dirichlet circular windows of small radii $a$ and $b$, separated by the Euclidean distance $\Delta=a+\Delta^{\prime}+b$ between their centers (see figure $6)$, we obtained for the NET $\bar{\tau}_{\varepsilon}[\mathbf{2 8}, \mathbf{2 7}]$ the expression

$$
\bar{\tau}_{\varepsilon}= \begin{cases}\frac{|\Omega|}{\pi D\left(\log \frac{1}{\varepsilon}+\log \frac{1}{\delta}\right)} \frac{\log \frac{1}{\delta} \log \frac{1}{\varepsilon}-\left[\log \left|\varepsilon+\Delta^{\prime}+\delta\right|+O(1)\right]^{2}}{1+2 \frac{\log \left|\varepsilon+\Delta^{\prime}+\delta\right|+O(1)}{\log \frac{1}{\delta}+\log \frac{1}{\varepsilon}}} & \text { for } \quad d=2 \\ \frac{|\Omega|}{4(a+b) D \tilde{r}} \frac{1-16 a b \tilde{r}^{2}\left(\frac{1}{2 \pi\left|a+\Delta^{\prime}+b\right|}+O(1)\right)^{2}}{1-\frac{8 a b \tilde{r}}{a+b}\left(\frac{1}{2 \pi\left|a+\Delta^{\prime}+b\right|}+O(1)\right)} & \text { for } \quad d=3,\end{cases}
$$

as $a, b, \varepsilon, \delta, \Delta^{\prime} \rightarrow 0$. Here $\tilde{r}=\tilde{r}\left(\Delta^{\prime}, \varepsilon, \delta\right)$ is a function of $\Delta^{\prime}, \varepsilon, \delta$ that varies monotonically between $\tilde{r}(0,0,0) \approx 0.6$ and $\tilde{r}\left(\Delta^{\prime}, 0,0\right) \rightarrow 1$ as $\Delta^{\prime} \rightarrow \infty$. These explicit dependence of the MFPT on the distance between the windows demonstrates that the result (1.21) is recovered as the windows drift apart and a new result is obtained as the windows touch (for $d=3$ ) or merge (for $d=2$ ). To derive these expressions, we have generalized the method of $[\mathbf{2 6}],[\mathbf{5 6}]-[\mathbf{5 8}]$, which consists in generalizing and solving Helmholtz's integral equation on the boundary, to the case of several separated or clustered windows. The asymptotic solution of the Helmholtz integral equation reveals the nonlinear interaction between the clustered windows. We will briefly illustrate in the next paragraph the method for the case of several windows. For $d=3$, it would be interesting to compute the log-singularity as discussed in the previous section.

Many targets. We shall describe here the general analysis for a large number of targets that can be clustered $[\mathbf{2 8}, \mathbf{2 7}]$. We consider an ensembles of circular 
Dirichlet windows $A_{i} \subset \partial \Omega,(i=1,2, \ldots, M)$, such that each has a neighbor separated from it by a distance comparable to their radii. The solution $u(\boldsymbol{x})$ of equation (1.10) with not forces $F=0$, has the following representation

$$
u(\boldsymbol{\xi})=\int_{\Omega} N(\boldsymbol{x}, \boldsymbol{\xi}) d \boldsymbol{x}+D \int_{\partial \Omega_{a}} N(\boldsymbol{x}, \boldsymbol{\xi}) \frac{\partial u(\boldsymbol{x})}{\partial n} d S_{\boldsymbol{x}}+C
$$

where $C$ is a constant to be determined from the boundary condition (1.11) and $d S \boldsymbol{x}$ is a surface area element on $\partial \Omega_{a}$. The condition that $u(\boldsymbol{x})$ vanishes on $\partial \Omega_{a}$ is

$$
F(\boldsymbol{\xi})=\sum_{i=1}^{M} \int_{A_{i}} N(\boldsymbol{x}, \boldsymbol{\xi}) g_{i}(\boldsymbol{x}) d S_{\boldsymbol{x}}, \quad \text { for all } \quad \boldsymbol{\xi} \in \partial \Omega_{a}=\bigcup_{i=1}^{M} A_{i}
$$

where

$$
F(\boldsymbol{\xi})=-\left(\int_{\Omega} N(\boldsymbol{x}, \boldsymbol{\xi}) d \boldsymbol{x}+C\right) .
$$

and the flux though window $A_{i}$ is $g_{i}(\boldsymbol{x})$. Summing the contributions of all fluxes (integrating equation (1.10) ), we get

$$
\sum_{i=1}^{M} \int_{A_{i}} g_{i}(\boldsymbol{x}) d S_{\boldsymbol{x}}=-|\Omega|
$$

To derive an expression for the mean time to a window, we shall compute the flux $g_{i}(\boldsymbol{x})$. For this, we derive a system of linear equations to solve. Each circular window $A_{i}$ is centered at $\mathbf{0}_{i}$ and has radius $\varepsilon_{i}$.

Using the explicit computation in dimension $3[\mathbf{2 8}]$, if $A, B$ denotes two windows, then

$$
\int_{A} \frac{g_{A}(\boldsymbol{x})}{\left|\boldsymbol{x}-\boldsymbol{x}^{\prime}\right|} d S_{\boldsymbol{x}}=\frac{1}{2 \pi} \varepsilon \tilde{g}_{A} \tilde{\alpha}(1+o(1)) \quad \text { for } \quad \boldsymbol{x}^{\prime} \in A, \quad \varepsilon<1
$$

where $\varepsilon$ is the radius of $\mathrm{A}$ and

$$
\int_{B} N\left(\boldsymbol{x}, \boldsymbol{x}^{\prime}\right) g_{B}(\boldsymbol{x}) d S \boldsymbol{x} \approx\left\{\begin{array}{l}
\frac{\tilde{\beta} \delta \tilde{g}_{B}(1+o(1))}{2 D \pi} \text { for } \quad \boldsymbol{x}^{\prime} \in B, \quad \delta<1 \\
\delta^{2} \tilde{g}_{B} \beta\left[N\left(\mathbf{0}_{A}, \mathbf{0}_{B}\right)+O(1)\right] \quad \text { for } \quad \boldsymbol{x}^{\prime} \in A, \quad \delta<1,
\end{array}\right.
$$

where $\beta$ and $\tilde{\beta}$ are constants. To obtain an expression for the flux, we use the asymptotic expansion

$$
g_{A}(\boldsymbol{x})=\frac{f_{\Delta}(\boldsymbol{x})}{\sqrt{1-\frac{|\boldsymbol{x}|^{2}}{\varepsilon^{2}}}}
$$

where $f_{\Delta}$ is of order one and normalized. Thus,

$$
\int_{A} g_{A}(\boldsymbol{x}) d S_{x}=\varepsilon^{2} \alpha \tilde{g}_{A}(1+o(1)), \quad \text { as } \quad \varepsilon, \rightarrow 0
$$


where $\alpha \approx 2 \pi$ and for $\boldsymbol{\xi}=\boldsymbol{\xi}_{j} \in A_{j}$, we obtain a system of $M+1$ linear equations for the unknown $\tilde{g}_{i}$ and $C$,

$$
\begin{aligned}
\frac{\pi}{2} \varepsilon_{j} \tilde{g}_{j}+\sum_{i \neq j}^{M} 2 \pi \varepsilon_{i}^{2} \tilde{g}_{i} N(i, j) & =F\left(\boldsymbol{\xi}_{j}\right) \approx-C, \quad j=1, \ldots, M, \\
2 \pi \sum_{i=1}^{M} \varepsilon_{i}^{2} \tilde{g}_{i} & =-|\Omega|,
\end{aligned}
$$

where $N(i, j)=N\left(\mathbf{0}_{i}, \mathbf{0}_{j}\right)$. Equation (1.41) is the flux condition. When all radii $\varepsilon_{i}$ can be scaled by $\varepsilon_{i}=\varepsilon \tilde{\varepsilon}_{i}$, where $\varepsilon=\min _{1 \leq i \leq M} \varepsilon_{i} \ll 1$, then for windows separated by distance $\Delta_{i, j}$,

$$
\max _{i, j} 2 \varepsilon N(i, j)=\max _{i, j} \frac{1}{\pi\left(\tilde{\varepsilon}_{i}+\tilde{\varepsilon}_{j}+\frac{\Delta_{i, j}}{\varepsilon}\right)}(1+o(1))<1 .
$$

Scaling $G_{j}=\pi \varepsilon_{j} \tilde{g}_{j} / C$, we write the symmetric matrix of the system (1.40) (with $1 / 2$ on the diagonal) as

$$
\boldsymbol{M}=\left(\begin{array}{cccc}
1 / 2 & 2 \tilde{\varepsilon}_{2} N(1,2) & \ldots & 2 \tilde{\varepsilon}_{M} N(1, M) \\
2 \tilde{\varepsilon}_{2} N(1,2) & . & . & . \\
\cdot & . & . & . \\
2 \tilde{\varepsilon}_{M} N(1, M) & \ldots & . & 1 / 2
\end{array}\right) .
$$

We decompose $\boldsymbol{M}$ as

$$
\boldsymbol{M}=\frac{1}{2} \boldsymbol{I}_{M}+\varepsilon \boldsymbol{A}
$$

where $\boldsymbol{I}_{M}$ is the identity matrix and $\boldsymbol{A}$ contains off-diagonal terms. Writing $\mathbf{1}_{M}$ (resp. $\tilde{\boldsymbol{G}}_{M}$ ) for a vector of 1 (respectively, of $G_{j}$ ), (1.40) becomes

$$
\left(\frac{1}{2} \boldsymbol{I}_{M}+\varepsilon \boldsymbol{A}\right) \tilde{\boldsymbol{G}}_{M}=-\mathbf{1}_{M}
$$

and can be inverted as the convergent series

$$
\tilde{\boldsymbol{G}}_{M}=-2 \sum_{k=0}^{\infty}(-2 \varepsilon \boldsymbol{A})^{k} \mathbf{1}_{M} .
$$

All terms can contribute to the sum, because $\varepsilon N(i, j)$ can be of order 1 . The interaction of the cluster with window $j$ is given by

$$
G_{j}=-2-2 \sum_{k=0}^{\infty}(-2 \varepsilon)^{k} \sum_{i_{1}, . ., i_{k}} N\left(j, i_{1}\right) N\left(i_{1}, i_{2}\right) \ldots N\left(i_{k-1}, i_{k}\right),
$$

where the sum is over all non-diagonal pairs (not all $i_{k}$ are different). The nonlinearity depends on the number of windows. In the first approximation,

$$
\tilde{\boldsymbol{G}}_{M} \approx-2\left(\boldsymbol{I}_{M}-2 \varepsilon \boldsymbol{A}\right) \mathbf{1}_{M}
$$


and

$$
\pi \frac{\varepsilon_{j} \tilde{g}_{j}}{C}=G_{j}=-2\left(1-2 \varepsilon \sum_{i \neq j} \tilde{\varepsilon}_{i} N(i, j)\right) .
$$

Using the condition (1.41), we obtain for the constant $C$ the equation

$$
-4 C \sum_{i=1}^{M} \varepsilon_{i}\left(1-2 \sum_{i \neq j} \varepsilon_{i} N(i, j)\right)=-|\Omega|,
$$

thus the NET through one of the window is

$$
C=\bar{\tau} \cup A_{i} \approx \frac{|\Omega|}{4 D} \frac{1}{\sum_{i=1}^{M} \varepsilon_{i}\left(1-2 \sum_{i \neq j} \varepsilon_{j} N(i, j)\right)} .
$$

It would be interesting to obtain an explicit expression 1.49 for disk uniformly distribution on a general domain. Other open directions are: what is the asymptotic as the number $\mathrm{N}$ becomes large for the NET in a domain with many holes, such that they occupied a small surface fraction $N \varepsilon^{2}=o(1)$. We would need to use all terms in the expansion 1.49. Depending on the windows organization and the initial Brownian particle location, this time does not necessarily tends to zero, as $\mathrm{N}$ goes to infinity (consider the case of an annulus with holes located only on the inner radius, while the Brownian particle starts on the outer-radius). Some analysis were presented in [5]. This problem requires a new analysis to treat the double asymptotic of large $N$ and small $\varepsilon$. Another direction is to study the NET for narrow domains [50]. In the remaining part of the review, we will focus on analyzing diffusion in the synaptic cleft, which is a specific a fundamental microdomains for neuronal communication.

\section{Diffusion in synapses, synaptic weight and synaptic transmission}

Despite of an active century of research in neuroscience, the brain remains a partially unknown and unpredictable machine. Several cellular populations participate to the brain function: inhibitory and excitatory neurons, glial and endothelial cells and many others. Neurons have been the most studied one and due to their complex excitable properties, they are considered to be the main device for processing information [32]. Neurons are organized in complex networks and functional areas that can sustain sophisticated brain functions. Neurons communicate through synapses and a single one can be connected to more than thousands of synapses, but two specific neurons may only have at most, less than ten connections between them $[\mathbf{6 2}]$.

Synapses are local active micro-contacts underlying direct neuronal communication but depending on the brain area where they are located and their specificity, they can vary in size and molecular composition. The molecular processes underlying synaptic transmission is well known [32]: after neurotransmitters such as glutamate, released from a vesicle located on the surface of a pre-synaptic neuron (see fig 3), they diffuse inside the synaptic cleft, composed of a pre and post synaptic terminal (fig.3). The post-synaptic terminal of excitatory synapses contains ionotropic glutamate receptors such as AMPA and NMDA receptors and they may 
open upon binding to neurotransmitters. AMPA receptors are tetrameric assemblies composed of four different subunits, which can bind to a glutamate molecule $[\mathbf{5 1}, \mathbf{1 8}]$. However, it has been reported that two glutamate molecules are required to open a single AMPA channel [31]. The amplitude of ionic current is thus proportional to the number of open receptors and their conductances. The postsynaptic current measures the efficiency of synaptic transmission and reports in a complex manner the frequency and location of released vesicles $[\mathbf{4 3}]$.

Due to the small synaptic cleft size, direct experimental recordings at individual synapses is still not possible. Thus, to study synaptic transmission, a first step was to study separately channels such as AMPARs, by expressing them in oocytes and by recording the current using patch-clamp and excised patch, where the conductance state properties related to the open, closed and desensitized states can been extracted by Markov chain models $[\mathbf{3 1}, \mathbf{3 8}, \mathbf{1 1}]$. However, to reduce the complexity of AMPA receptor dynamics, Markov chains were kept as minimal as possible to account for one or two possible binding sites $[\mathbf{3 1}, \mathbf{3 8}, \mathbf{1 1}]$. Only recently $[48]$, to study the high variability $(\sim 5-100 \mathrm{pA})$ of the synaptic current $I_{s}$, a four state channel model was used to interpret the current high coefficient of variation, which was shown to be due to the spatio-temporal correlations of two released vesicles.

Once receptor properties were sufficiently characterized, a second step in synaptic studies consisted in integrating the local and molecular properties within the synaptic organization to identify the entire synaptic function. This step became possible by the use of modeling and numerical simulations $[48,29,3,17,53]$ and resulted in estimating the role of synaptic geometry on the number of open receptors. More recently using the properties of glutamate diffusion and the electrical resistance inside the synaptic cleft, it was anticipated that the synaptic current is maximal for an optimal cleft height [53].

We review here some properties of synaptic transmission that were brought by our recent study based on diffusion in microdomains. Contrary to previous approaches, based on a molecular biophysical analysis of diffusion in the synaptic cleft, that we will present here, we estimated the mean and the variance of the postsynaptic current. This current plays a fundamental role in neuronal communication: it is the direct and fast signal of synaptic transmission. It can activate the postsynaptic neuron or modulate its membrane potential. This current depends on receptors such as AMPA and the complex molecular machinery edifice underneath controlling them. Finally, possible changes in the current dynamics is the read out of synaptic plasticity, a process that underlies learning and memory [43].

Modeling the synaptic current. In [66], we modeled glutamates as diffusing particles and approximated the cleft geometry as a narrow cylinder [7]. Instead of using the classical Markov description $[\mathbf{3 1}, \mathbf{3 8}, \mathbf{1 1}]$, obtained by optimal fitting of measurements performed outside a synapse, our analysis $[\mathbf{6 6}]$ relied on the direct analysis of AMPA conductances [18] and we accounted for the four glutamate binding sites per receptor. While glutamate molecules are Brownian inside the cylindrical synaptic cleft $\Omega$, AMPARs are positioned on the PSD. We fixed their number to $N_{a}$. To model glutamate binding to an AMPAR, we used on the PSD $\left(\Omega_{P S D}\right)$ a radiative boundary condition $[\mathbf{7 4}, \mathbf{4}]$, that will be discussed later on. In addition, when a glutamate hits the lateral boundary $\left(\partial \Omega_{L a t}\right)$ of the cleft cylindrical domain, it is absorbed and thus cannot contribute anymore to AMPAR's activation. The probability density function $p\left(\boldsymbol{x}, t \mid \boldsymbol{x}_{0}\right)$ that a glutamate molecule is located at 
position $\boldsymbol{x}$ at time, when it started at position $\boldsymbol{x}_{0}$ satisfies the diffusion equation:

$$
\begin{aligned}
\frac{\partial p\left(\boldsymbol{x}, t \mid \boldsymbol{x}_{0}\right)}{\partial t} & =D \Delta p\left(\boldsymbol{x}, t \mid \boldsymbol{x}_{0}\right), \quad \boldsymbol{x} \in \Omega, t>0 \\
p(\boldsymbol{x}, 0) & =\delta\left(\boldsymbol{x}-\boldsymbol{x}_{0}\right) \\
\left.\frac{\partial p\left(\boldsymbol{x}, t \mid \boldsymbol{x}_{0}\right)}{\partial \nu}\right|_{\partial \Omega_{r}}=0, & \left.p\left(\boldsymbol{x}, t \mid \boldsymbol{x}_{0}\right)\right|_{\partial \Omega_{\text {Lat }}}=0 \\
-\left.D \frac{\partial p\left(\boldsymbol{x}, t \mid \boldsymbol{x}_{0}\right)}{\partial \nu}\right|_{\partial \Omega_{P S D}} & =-\kappa p\left(\boldsymbol{x}, t \mid \boldsymbol{x}_{0}\right),
\end{aligned}
$$

where $D$ is the free glutamate diffusion constant. The partial absorption constant $\kappa$ not only accounts for the fraction of AMPARs inside the PSD, but also for the activation barrier of a single glutamate to a glutamate receptor binding site. We have proposed [66] the following expression:

$$
\kappa=\frac{D}{2 \pi R_{P S D}^{2}} \frac{1}{\frac{1}{N_{a} a}+\frac{D}{\kappa_{e} 2 \pi a^{2} N_{a}}}
$$

where $a$ and $R_{P S D}$ are the radius of a single receptor and the PSD respectively and $N_{a}$ is the number of AMPARs.

The probability $p\left(\boldsymbol{x}_{0}\right)$ that a glutamate molecule binds a receptor, when it is released from a position $\boldsymbol{x}_{0}$, is given by the total flux:

$$
(1.52) p\left(\boldsymbol{x}_{0}\right)=D \int_{0}^{\infty} \int_{\partial \Omega_{P S D}} \frac{\partial p\left(\boldsymbol{y}, t \mid \boldsymbol{x}_{0}\right)}{\partial n} d \boldsymbol{y} d t=D \int_{\partial \Omega_{P S D}} \frac{\partial u\left(\boldsymbol{y} \mid \boldsymbol{x}_{0}\right)}{\partial n} d \boldsymbol{y}
$$

where $u\left(\boldsymbol{x} \mid \boldsymbol{x}_{0}\right)=\int_{0}^{\infty} p\left(\boldsymbol{x}, t \mid \boldsymbol{x}_{0}\right) d t$ satisfies

$$
\begin{aligned}
& D \Delta u\left(\boldsymbol{x} \mid \boldsymbol{x}_{0}\right)=-\delta\left(\boldsymbol{x}-\boldsymbol{x}_{0}\right) \text { for } \quad \boldsymbol{x} \in \Omega \\
&\left.\frac{\partial u\left(\boldsymbol{x} \mid \boldsymbol{x}_{0}\right)}{\partial \nu}\right|_{\partial \Omega_{r}}=0,\left.u\left(\boldsymbol{x} \mid \boldsymbol{x}_{0}\right)\right|_{\partial \Omega_{L a t}}=0 \\
&\left.D \frac{\partial u\left(\boldsymbol{x} \mid \boldsymbol{x}_{0}\right)}{\partial \nu}\right|_{\partial \Omega_{P S D}}=\kappa u\left(\boldsymbol{x} \mid \boldsymbol{x}_{0}\right),
\end{aligned}
$$

In the next paragraph, we present the analytical expressions for $u$ and $p\left(\boldsymbol{x}_{0}\right)$ that we use to obtain numerical simulations. These expressions serve to calibrate the partial absorbing constant $\kappa_{e}$ in eq.(1.51), such that in the range from one to four vesicles, the number of activated AMPARs was not saturating (see figure 7). For a synaptic radius of $0.5 \mu \mathrm{m}$, a PSD radius of $0.3 \mu \mathrm{m}$ and a height of $0.02 \mu \mathrm{m}$. We found by plotting the associated current (see figure 7 ) that $\kappa_{e} \approx 1.6$.

The mean and variance of the synaptic current $I_{s}$. To compute the mean and the variance of the synaptic current, we account for the following two stochastic components: the number of bound glutamate molecules and the probability of a given configuration of bound AMPARs. To estimate the first, we recall that the probability distribution $\operatorname{Pr}_{k}\left(\boldsymbol{x}_{0}\right)$ to have $k$ independent glutamate molecules bound, when a vesicle is released at position $\boldsymbol{x}_{0}$ follows a binomial distribution

$$
\operatorname{Pr}_{k}\left(\boldsymbol{x}_{0}\right)=C_{4 N_{a}}^{k} p\left(\boldsymbol{x}_{0}\right)^{k}\left(1-p\left(\boldsymbol{x}_{0}\right)\right)^{4 N_{a}-k}
$$



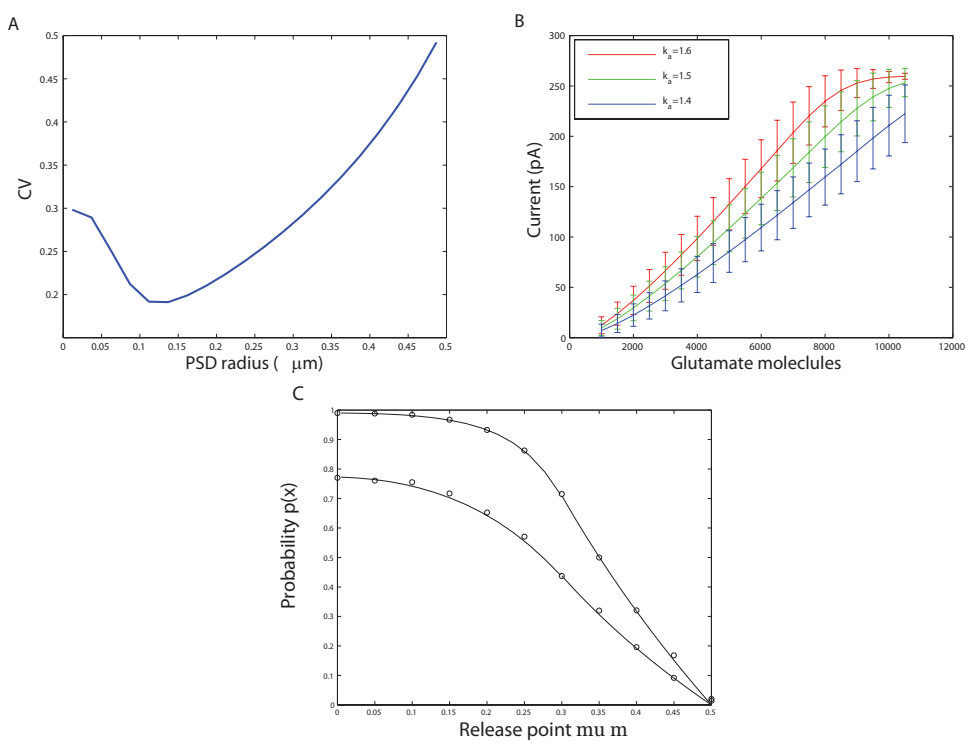

FigURE 7. A: Optimal PSD radius: For a PSD radius of $0.3 \mu m$, the coefficient of variation $(\mathrm{CV})$ reaches a maximum. This shows that for a given size of the active zone, where vesicles are released, the CV is minimum when receptors are spread over an optimal PSD area. B: Calibration the partial absorbing constant $\kappa$. We simulate the synaptic current using eq. 1.56 for different values of the activation coefficient $k$ (which enters into the probability $P r_{q}$ of equation 1.53. The optimal synaptic response in the range of 3,00012,000 glutamate molecules and it corresponds to $\kappa=1.6$. C: we plotted the probability $p\left(\boldsymbol{x}_{0}\right)$ (eq.1.74) and compare the analytical curves (1.74) (continuous line) with Brownian simulations (dots) obtained with the partial reflective scheme 1.76 in the cylindrical domain of radius $R=0.5$, height 0.02 . The radius of the PSD is $L=0.3$.

where $N_{a}$ is the mean number of bound glutamates and the probability $p\left(\boldsymbol{x}_{0}\right)$ that a glutamate binds one of the receptors before it escapes is given by equation (1.52)

$$
p\left(\boldsymbol{x}_{0}\right)=\kappa \int_{\partial \Omega_{P S D}} u\left(\boldsymbol{x} \mid \boldsymbol{x}_{0}\right) d S(\boldsymbol{x})
$$

The mean and the variance of the number of bound receptors are given respectively by $M\left(\boldsymbol{x}_{0}\right)=N_{g} p\left(\boldsymbol{x}_{0}\right), \sigma^{2}\left(\boldsymbol{x}_{0}\right)=N_{g} p\left(\boldsymbol{x}_{0}\right)\left(1-p\left(\boldsymbol{x}_{0}\right)\right)$. When the vesicle is released at the center of the synapse, for a PSD and a synaptic radius of respectively $300 \mathrm{~nm}$ and $500 \mathrm{~nm}$ (the effective surface area of a single AMPAR is $10 \mathrm{~nm}^{2}$ and $\kappa=0.25$ (see fig.(7)), using equations (1.72),(1.73),(1.74),(1.75), we obtain for a glutamate to hit a cluster of AMPARs, a probability of $p\left(\boldsymbol{x}_{0}\right)=8.10^{-3}$.

The second source of fluctuation is the following: AMPARs can be bound to various glutamate (from zero to four), thus the probability of a given configuration $\vec{n}=\left(n_{4}, n_{3}, n_{2}, n_{1}\right)$ to have $n_{1}$ AMPARs bound to 1 glutamate, $n_{2}$ AMPARs bound to 2 glutamates and so on, when there are $N_{a}$ AMPA receptors, for $k<4 N_{a}$ bound 
glutamate molecules, is given by

$$
\operatorname{Pr}\{\vec{n} \mid k\}=\frac{N_{a} !}{n_{4} ! n_{3} ! n_{2} ! n_{1} !\left(N_{a}-\left(n_{4}+n_{3}+n_{2}+n_{1}\right)\right) !} \frac{1}{F\left(k, N_{a}\right)}
$$

which corresponds of choosing $n_{4}$ AMPARs out of $N_{4}, n_{3}$ out of $N_{a}-n_{4}$ and so on. $F\left(k, N_{a}\right)$ is the total number of possibilities to decompose the number $k$ as a sum of $N_{a}$ on the integer $4,3,2,1,0$. In practices, we compute $F\left(k, N_{a}\right)$ numerically as the $k+1$ 's coefficient of the expression $\left(1+x+x^{2}+x^{3}+x^{4}\right)^{N_{a}}$. The present analysis can be used to obtain any statistical moments associated with the current and in particular, the mean and variance are given by

$$
\begin{aligned}
<I_{s}\left(\boldsymbol{x}_{0}\right)>= & \sum_{k=1}^{N_{g}} \sum_{n \in S_{k}} \vec{n} \cdot \vec{\gamma} \operatorname{Pr}\{\vec{n} \mid k\} V \operatorname{Pr}_{k}\left(\boldsymbol{x}_{0}\right)= \\
& \sum_{k=1}^{4 N_{a}} \sum_{n \in S_{k}} \vec{n} \cdot \vec{\gamma} \operatorname{Pr}\{\vec{n} \mid k\} \operatorname{Pr}_{k}\left(\boldsymbol{x}_{0}\right) V+ \\
& N_{a} \gamma_{4} V\left(1-\sum_{k=0}^{4 N_{a}} \operatorname{Pr}_{k}\left(\boldsymbol{x}_{0}\right)\right) \\
<I_{s}^{2}\left(\boldsymbol{x}_{0}\right)>= & \sum_{k=1}^{N_{g}} \sum_{n \in S_{k}}(\vec{n} \cdot \vec{\gamma})^{2} \operatorname{Pr}\{\vec{n} \mid k\} \operatorname{Pr}_{k}\left(\boldsymbol{x}_{0}\right) V^{2}-<I_{s}\left(\boldsymbol{x}_{0}\right)>^{2} \\
& \sum_{k=1}^{4 N_{a}} \sum_{n \in S_{k}}(\vec{n} \cdot \vec{\gamma} V)^{2} \operatorname{Pr}\{\vec{n} \mid k\} \operatorname{Pr}_{k}\left(\boldsymbol{x}_{0}\right)+ \\
& \left(N_{a} \gamma_{4} V\right)^{2}\left(1-\sum_{k=0}^{4 N_{a}} \operatorname{Pr}_{k}\left(\boldsymbol{x}_{0}\right)\right)-<I_{s}\left(\boldsymbol{x}_{0}\right)>^{2}
\end{aligned}
$$

where $\mathrm{V}$ is the potential and $\vec{\gamma}=\left(\gamma_{1}, \gamma_{2}, \gamma_{3}, \gamma_{4}\right)$ is the conductance vector, $S_{k}$ are all the possible configurations of $\vec{n}=\left(n_{1}, n_{2}, n_{3}, n_{4}\right)$ such that $4 n_{4}+3 n_{3}+2 n_{2}+n_{1}=$ $k$. The formula for the mean and variance are composed of two terms: in the first one, we sum over sites that are partially bounded by glutamate molecules. The probability for such an event is the product of the probability $P r_{k}$, that $k$ glutamates are bound $\left(k<4 N_{a}\right)$ and the probability $\operatorname{Pr}\{\vec{n} \mid k\}$ for a given binding configuration $k=4 n_{4}+3 n_{3}+2 n_{2}+n_{1}$. The second term accounts for the event where all AMPAR binding sites are occupied $\left(4 N_{a}\right)$, the probability is the complementary to the one associated with the partial binding of the first case.

Solution of the Laplace equation in a narrow domain: reduction dimension by the method of averaging. We present here the averaging method we used to obtain an expression for the probability $p\left(\boldsymbol{x}_{0}\right)$ that we will use later on to obtain numerical estimates. To that end, we solve the steady state diffusion equation associated to equation 1.50 in the cylindrical cleft geometry and when 
glutamates are released at a point $\boldsymbol{x}_{0}$. We start with the Laplace equation

$$
D \Delta u(r, z)=-\delta(r) \delta\left(z-z_{0}\right) \quad \text { for } \quad\{(r, z) \mid r \in[0, R), z \in(0, h)\}
$$

$$
\begin{gathered}
\left.\frac{\partial u(r, z)}{\partial \nu}\right|_{\partial \Omega_{r}}=0,\left.\quad u(r, z)\right|_{r=R}=0 \\
\left.D \frac{\partial u(r, z)}{\partial \nu}\right|_{r<L, z=0}=\kappa u(r, z),
\end{gathered}
$$

In cylindrical coordinates, the average $\bar{u}(r)=\frac{1}{h} \int_{0}^{h} u(r, z) d z$ satisfies

$$
D\left(\bar{u}^{\prime \prime}(r)+\frac{1}{r} \bar{u}^{\prime}(r)-\frac{1}{h} \frac{\partial}{\partial z} u(r, 0) \chi_{[0, L]}(r)\right)=-\frac{1}{r h} \delta\left(r-r_{0}\right),
$$

Using the boundary conditions on $\partial \Omega_{P S D}$ for $r<L, z=0$, we express $\left.\frac{\partial}{\partial z} u(r, 0)\right) \chi_{[0, L]}(r)=$ 0 in terms of $\bar{u}$ :

$$
u(r, z) \approx u(r, 0)+\frac{\partial u(r, 0)}{\partial z} z+O\left(h^{2}\right)
$$

Integrating the Taylor expansion with respect to $z$, and using that for $u(r, 0)=$ $\frac{D}{\kappa} \frac{\partial u(r, 0)}{\partial z}$ for $r<L$,

$$
\bar{u} \approx \frac{\partial u(r, 0)}{\partial z}\left(\frac{D}{\kappa}+\frac{h}{2}\right),
$$

by substituting eq.(1.59) in (1.58), we get

$$
D\left(\bar{u}^{\prime \prime}(r)+\frac{1}{r} \bar{u}^{\prime}(r)-\frac{2 \kappa}{h(2 D+\kappa h)} \bar{u} \chi_{[0, L]}(r)\right)=-\frac{1}{r h} \delta\left(r-r_{0}\right) .
$$

The solution can be expressed in terms of the Bessel function $K_{0}$ and $I_{0}$

$$
\bar{u}(r)=\left\{\begin{array}{cl}
\frac{1}{2 \pi D} K_{0}(\alpha r)+A I_{0}(\alpha r) & 0<r<L \\
C \log \frac{r}{R} & L<r<R
\end{array},\right.
$$

where

$$
\alpha=\sqrt{\frac{2 \kappa}{h(2 D+\kappa h)}} .
$$

To determine the parameters $A$ and $C$, we use the continuity of $\bar{u}$ and its derivative at $r=L$. We obtain the linear system to invert:

The solution is given by

$$
\begin{aligned}
& \frac{1}{D} K_{0}(\alpha L)+A I_{0}(\alpha L)=C \log \frac{L}{R} \\
& -\frac{1}{D} \alpha K_{1}(\alpha L)+A \alpha I_{1}(\alpha L)=C \frac{1}{L} .
\end{aligned}
$$

$$
\begin{aligned}
& \left(\begin{array}{c}
A \\
C
\end{array}\right)=\left(\begin{array}{cc}
\alpha I_{0}(\alpha L) & -\log \frac{L}{R} \\
\alpha I_{1}(\alpha L) & \frac{1}{L}
\end{array}\right)^{-1}\left(\begin{array}{c}
-\frac{1}{D} K_{0}(\alpha L) \\
\frac{1}{D} \alpha K_{1}(\alpha L)
\end{array}\right)= \\
& \frac{1}{D \alpha\left(\log \frac{L}{R} I_{1}(\alpha L)-L^{-1} I_{0}(\alpha L)\right)}
\end{aligned}\left(\begin{array}{c}
L^{-1} K_{0}(\alpha L)+\log \frac{L}{R} K_{1}(\alpha L) \\
\alpha I_{1}(\alpha L) K_{0}(\alpha L)+\alpha I_{0}(\alpha L) K_{1}(\alpha L)
\end{array}\right)
$$


To compute the probability $p\left(\boldsymbol{x}_{0}\right)$ in Eq.(1.54), we need to estimate two fluxes: first at the PSD given by

$$
\begin{aligned}
J_{P S D} & =\kappa \int_{\partial \Omega_{P S D}} u(P) d S_{P} \\
& =2 \pi \kappa \int_{0}^{L} \frac{1}{2 \pi D} K_{0}(\alpha r) r d r+2 \pi \kappa \int_{0}^{L} A I_{0}(\alpha r) r d r
\end{aligned}
$$

and second the flux at the lateral boundary

$$
J_{L a t}=-2 \pi D R h \bar{u}^{\prime}(R)=-2 \pi D h C .
$$

Thus when the vesicle is released at the center, the probability to hit one of the receptor is given by

$$
\begin{aligned}
p & =\frac{J_{P S D}}{J_{P S D}+J_{L a t}} \\
& =\frac{2 \pi \kappa \int_{0}^{L} \frac{1}{2 \pi D} K_{0}(\alpha r) r d r+2 \pi \kappa \int_{0}^{L} A I_{0}(\alpha r) r d r}{2 \pi \kappa \int_{0}^{L} \frac{1}{2 \pi D} K_{0}(\alpha r) r d r+2 \pi \kappa \int_{0}^{L} A I_{0}(\alpha r) r d r-2 \pi D h C}
\end{aligned}
$$

This expression will be used to compute numerically $p(0)$.

Estimation of the probability for a general location of a vesicle release. To estimate the flux for a general location of vesicular release, we use the general expression of the Laplacian (1.57),

$$
\begin{aligned}
& \frac{\partial^{2} \bar{u}(r, \theta)}{\partial r^{2}}+\frac{1}{r} \frac{\partial \bar{u}(r, \theta)}{\partial r}+\frac{1}{r^{2}} \frac{\partial^{2} \bar{u}(r, \theta)}{\partial^{2} \theta}-\alpha^{2} \bar{u}(r, \theta) \chi_{[0, L]} \\
= & -\frac{1}{r h D} \delta\left(r-r_{0}\right) \delta\left(\theta-\theta_{0}\right)
\end{aligned}
$$

We chose the release point on the line $\theta_{0}=0$, the solution can be developed in a cosine series

$$
\bar{u}(r \theta)=\frac{a_{0}}{2}+\sum_{n=1}^{\infty} a_{n} \cos (n \theta)
$$

where the $a_{n}$ might depend on $n$. To estimate the flux, we compute

$$
\kappa \int_{0}^{2 \pi} \int_{0}^{L} \bar{u}(r, \theta) r d r d \theta=\kappa \int_{0}^{2 \pi} \int_{0}^{L} \frac{a_{0}\left(r, r_{0}\right)}{2} r d r d \theta
$$

By substituting the expansion (1.66) in equation(1.65) and integrating with respect to $\theta$ we found that $a_{0}$ satisfies

$$
\frac{d^{2} a_{0}\left(r \mid r_{0}\right)}{d r^{2}}+\frac{1}{r} \frac{d a_{0}\left(r \mid r_{0}\right)}{d r}-\alpha^{2} a_{0}\left(r \mid r_{0}\right) \chi_{[0, L]}=\frac{1}{D \pi h r} \delta\left(r-r_{0}\right)
$$

the solution has the form

$$
a_{0}(r)=\left\{\begin{array}{cc}
A_{1} I_{0}(\alpha r) & 0<r \leq r_{0} \\
A_{2} I_{0}(\alpha r)+B_{2} K_{0}(\alpha r) & r_{0}<r \leq L \\
A_{3} \log (r / R) & L<r<R
\end{array}\right.
$$

When $r_{0}<L$, the continuity at the point $\boldsymbol{x}_{0}=\left(r_{0}, \theta_{0}=0\right)$ leads to

$$
A_{1} I_{0}(\alpha r)-A_{2} I_{0}\left(\alpha r_{0}\right)-B_{2} K_{0}\left(\alpha r_{0}\right)=0
$$


while integrating equation(1.67) over $\left(r_{0}-\varepsilon, r_{0}+\varepsilon\right)$, we obtain the condition

$$
\int_{r_{0}-\varepsilon}^{r_{0}+\varepsilon} \frac{d}{d r}\left(r \frac{d a_{0}\left(r \mid r_{0}\right)}{d r}\right) d r-\int_{r_{0}-\varepsilon}^{r_{0}+\varepsilon} \alpha^{2} a_{0}\left(r \mid r_{0}\right) r d r=\frac{1}{D \pi h}
$$

Taking the limit $\varepsilon \rightarrow 0$, we get that

$$
A_{2} I_{1}\left(\alpha r_{0}\right)-B_{2} K_{1}\left(\alpha r_{0}\right)-A_{1} I_{1}\left(\alpha r_{0}\right)=\frac{1}{\alpha D \pi h r_{0}}
$$

The final condition comes from the interface $r=L$, where we require the continuity of $a_{0}$ and its derivative with respect to $r$. We get

$$
\begin{array}{r}
A_{2} I_{0}(\alpha L)+B_{2} K_{0}(\alpha L)-A_{3} \log (L / R)=0 \\
A_{2} I_{1}(\alpha L)-B_{2} K_{1}(\alpha L)-A_{3} \frac{1}{\alpha L}=0
\end{array}
$$

From equations (1.68-1.71), we obtain four independent equations for the coefficients $A_{1}, A_{2}, B_{2}, A_{3}$ and the net flux can be expressed as

$$
\begin{aligned}
& J_{P S D}\left(\boldsymbol{x}_{0}\right)=\kappa \int_{\partial \Omega_{P S D}} u\left(r \mid \boldsymbol{x}_{0}\right) d S \\
& =\kappa \int_{0}^{r_{0}}\left(I_{0}(\alpha r), 0,0,0\right)\left(\begin{array}{cccc}
I_{0}(\alpha r) & -I_{0}\left(\alpha r_{0}\right) & -K_{0}\left(\alpha r_{0}\right) & 0 \\
-I_{1}\left(\alpha r_{0}\right) & I_{1}\left(\alpha r_{0}\right) & -K_{1}\left(\alpha r_{0}\right) & 0 \\
0 & I_{0}(\alpha L) & K_{0}(\alpha L) & -\log (L / R) \\
0 & I_{1}(\alpha L) & -K_{1}(\alpha L) & -\frac{1}{\alpha L}
\end{array}\right)^{-1}\left(\begin{array}{c}
0 \\
\frac{1}{\alpha D} \\
0 \\
0
\end{array}\right) r d r+ \\
& \kappa \int_{r_{0}}^{L}\left(0, I_{0}(\alpha r), K_{0}((k \text { r } \%) 0)\left(\begin{array}{cccc}
I_{0}(\alpha r) & -I_{0}\left(\alpha r_{0}\right) & -K_{0}\left(\alpha r_{0}\right) & 0 \\
-I_{1}\left(\alpha r_{0}\right) & I_{1}\left(\alpha r_{0}\right) & -K_{1}\left(\alpha r_{0}\right) & 0 \\
0 & I_{0}(\alpha L) & K_{0}(\alpha L) & -\log (L / R) \\
0 & I_{1}(\alpha L) & -K_{1}(\alpha L) & -\frac{1}{\alpha L}
\end{array}\right)^{-1}\left(\begin{array}{c}
0 \\
\frac{1}{\alpha D} \\
0 \\
0
\end{array}\right) r d r\right. \text {. }
\end{aligned}
$$

In addition, the lateral flux is given by

$$
J_{\text {Lat }}\left(\boldsymbol{x}_{0}\right)=-2 \pi D R h \bar{u}^{\prime}(R)=-2 \pi D h A_{3}
$$

Thus the total probability for one receptor to be activated when the neurotransmitters are released at position $\boldsymbol{x}_{0}$, is given by

$$
p\left(\boldsymbol{x}_{0}\right)=\frac{J_{P S D}\left(\boldsymbol{x}_{0}\right)}{J_{P S D}\left(\boldsymbol{x}_{0}\right)+J_{L a t}\left(\boldsymbol{x}_{0}\right)} .
$$

In practice, to compute (1.74), we solve (1.68-1.71) numerically.

When a vesicle is released at a position outside the PSD, $\left.r_{0} \geq L\right), a_{0}$ has the form

$$
a_{0}(r)=\left\{\begin{array}{cc}
A_{1} I_{0}(\alpha r) & 0<L \leq r_{0} \\
A_{2} \log (r)+B_{2} & L<r \leq r_{0} \\
A_{3} \log (r / R) & r_{0}<r<R .
\end{array}\right.
$$


Using similar considerations as previously, the continuity conditions lead to the set of equations:

$$
(*)\left\{\begin{array}{ccc}
A_{1} I_{0}(\alpha L)-A_{2} \log (L)-B 2 & =0 \\
A_{1} \alpha I_{1}(\alpha L)-\frac{A_{2}}{L} & =0 \\
A_{2} \log \left(r_{0}\right)+B_{2}-A_{3} \log \frac{r_{0}}{R} & =0 \\
-\frac{A_{2}}{r_{0}}+\frac{A_{3}}{r_{0}} & = & \frac{-1}{\pi D h r_{0}}
\end{array}\right.
$$

Solving the linear system $(*)$, we obtain the following expression for the flux:

$$
J_{P S D}\left(\boldsymbol{x}_{0}\right)=\int_{0}^{L}\left(I_{0}(\alpha r), 0,0,0\right)\left(\begin{array}{cccc}
I_{0}(\alpha L) & -\log (L) & -1 & 0 \\
\alpha I_{1}(\alpha L) & \frac{-1}{L} & 0 & 0 \\
0 & \log \left(r_{0}\right) & 1 & -\log \frac{r_{0}}{R} \\
0 & -\frac{1}{r_{0}} & 0 & \frac{1}{r_{0}}
\end{array}\right)^{-1}\left(\begin{array}{c}
0 \\
0 \\
0 \\
\frac{-1}{D h r_{0}}
\end{array}\right) r d r
$$

Since our analytical analysis contains several approximations such as the averaging over the cleft height, in addition to use numerical methods to compute explicitly the coefficients $A_{1}, A_{2}, B_{2}, A_{3}$, we evaluate the accuracy of our analysis by comparing the analytical formula for the probability (1.74) with Brownian simulations (Fig. 7b). We simulated Brownian particles in the same cylindrical domain as the one used for the analytical computation with the absorbing lateral boundary. We put at the PSD a partial absorbing boundary with the condition $-D \frac{\partial p}{\partial n}=-\kappa p$. At the particle level, we implemented a reflection (fig. 7) rule [61], in which the particle hitting the PSD boundary is reflected with a probability

$$
P=\kappa \frac{\sqrt{\pi}}{\sqrt{D}}
$$

where $D$ is the diffusion constant and $\Delta t$ is the time step of the simulation. The scheme is standard when the glutamate molecule is inside the cleft, but when $x(t)+$ $\sqrt{2 D} \Delta w<0$ at the PSD, then we reflect according to $[\mathbf{6 1}]$

$$
x(t+\Delta t)=\left\{\begin{array}{l}
-(x(t)+\sqrt{2 D} \Delta w) \quad \text { w.p. } 1-P \sqrt{\Delta t} \\
\text { terminate trajectory otherwise. }
\end{array}\right.
$$

Final remarks and conclusion. We found here that the synaptic current $I_{s}$ depends on the vesicular release location, the number and the biophysical properties of the receptors, the PSD size and location, and the geometrical characteristic of the synaptic cleft. We use a calibration procedure to find $\kappa=1.6$, this value gives the largest variable response for a range of one to three released vesicles. Furthermore, our analysis reveals that given the pre-synaptic active zone size, where vesicles are released, the synaptic current is maximal as long as the PSD size remains smaller than the active zone radius. Change in the PSD size can be mediated by synaptic remodeling processes. Remodeling the PSD can be a source of synaptic plasticity and modulation for synaptic transmission and these changes can be mediated by geometrical re-organization of the synaptic geometry, or internal scaffolding reorganization $[\mathbf{1 5}, \mathbf{6}]$. These changes can thus affect the detection threshold of the post-synaptic neuron $[\mathbf{1 3}, \mathbf{1 4}, \mathbf{1 5}]$ and be a direct source of synaptic plasticity without affecting the number of AMPA receptors.

The synapse is specific a microdomain, in which the theory of diffusion has already revealed some of the basic properties such as the time scale and the number of activated receptors, the effect of the geometry and a possible optimal size of the 
PSD that leads to a reliable synaptic current. Together with precise molecular information, future works should include the precise mechanisms underlying synaptic plasticity and transmission in normal and pathological conditions.

Finally, because the post-synaptic terminal of excitatory neurons ends on dendritic spines rather than on the dendritic shaft, it would be very interesting to extend previous models $[\mathbf{2 3}, \mathbf{2 4}, \mathbf{2 2}]$ to include several signaling pathways involved in synaptic plasticity. Indeed, dendritic spines concentrate molecules involved in calcium regulation and synaptic plasticity. The precise regulation by calcium and leading to a change in the synaptic current is still unclear.

Historical note: In 2002, I decided to model cellular microdomains and receptor trafficking while I was at UCSF (University of California at San Francisco). The escape time of a receptor from a synaptic micro-domain was a question of interest for synaptic transmission, especially after Daniel Choquet's 2002 paper. With Zeev Schuss, we started working on estimating the resident time of a receptor at a synapse, which provides specific information about synaptic sensitivity to trafficking. We first developed this idea in the [26]. Later on, with Amit Singer, we extended the analysis in the 3 papers under the name of "narrow escape" $[\mathbf{5 6}, \mathbf{5 7}, \mathbf{5 8}]$. It was only in 2006 that we learnt from an anonymous reviewer about the early analysis, during the 1990's, of M. Ward and J. Keller, using matching asymptotic $[\mathbf{7 0}, \mathbf{7 1}, \mathbf{7 2}, \mathbf{3 5}]$. We recently extended the narrow escape time to a polymer to a small hole, potential with trapped and many other directions. We hope that this research direction will continue attracting the attention of cellular biologists, biophysicists, physicists, neuroscientists and applied mathematicians interested in deriving cellular principles and functions from the molecular level. The second part of this review is actually the third part of A. Taflia $\mathrm{PhD}$ thesis.

\section{References}

[1] Armstrong, N. Sun, Y. Chen. G. Q. Gouaux, E. (1998) Structure of a glutamate-receptor ligand-binding core in complex with kainate.Nature. 395(6705), pp. 9137.

[2] Armstrong, N. Jasti, J. Beich-Frandsen, M. and Gouaux, E. (2006) Measurement of conformational changes accompanying desensitization in an ionotropic glutamate receptor. Cell.127 (1), pp. 8597.

[3] Barbour, B. (2001) An evaluation of synapse independence. J. Neur.21(20), pp. 7969-84.

[4] Berezhkovskii, A. Makhnovskii, Y. Monine M. Zitserman V. and Shvartsman, S. (2004) Boundary homogenization for trapping by patchy surfaces.J. Chem. Phys. 121(22), pp. 11390-4.

[5] H.C. Berg and E.M. Purcell, "Physics of chemoreception", Biophys. J. 20 pp.193-219 (1977).

[6] A. Blanpied, J. M. Kerr and M. D. Ehlers, (2008) Structural plasticity with preserved topology in the postsynaptic protein network PNAS. 105(34), pp. 12587-12592.

[7] Bourne, J. N. Harris, K. M. (2008) Balancing Structure and Function at Hippocampal Dendritic Spines. Ann. Rev. Neur. 31, pp. 47-67.

[8] Borgdorff AJ, Choquet D. Regulation of AMPA receptor lateral movements. Nature. 2002; 417(6889):649-53.

[9] Bredt, D. S. and Nicoll, R. A. (2003) AMPA receptor trafficking at excitatory synapses. Neuron.40(2), pp. 361-79.

[10] Choquet D, Triller A (2003) The role of receptor diffusion in the organization of the postsynaptic membrane. Nature Rev Neurosci :251-65.

[11] Cho, C. H. St-Gelais. F. Zhang, W. Tomita, S. and Howe, J. R. (2007) Two families of TARP isoforms that have distinct effects on the kinetic properties of AMPA receptors and synaptic currents. Neuron. 55(6), pp. 890-904.

[12] Cingolani, L. A. Goda Y. (2008) Actin in action: the interplay between the actin cytoskeleton and synaptic efficacy. Nat. Rev. Neur. 9(5), pp. 344-56. 
[13] Elias, G. M. and Nicoll, R. A. (2007) Synaptic trafficking of glutamate receptors by MAGUK scaffolding proteins. Trends Cell. Biol. 17(7), pp. 343-352.

[14] Elias, G. M. et. al. (2006) Synapse-specific and developmentally regulated targeting of AMPA receptors by a family of MAGUK scaffolding proteins. Neuron. 52(2), pp. 307-320.

[15] El-Husseini, A. E. Schnell, E. Chetkovich, D. M. Nicoll, R. A. and Bredt D. S. (2000) PSD-95 involvement in maturation of excitatory synapses. Science. 290(5495), pp. 1364-8.

[16] V.I. Fabrikant, Mixed Boundary Value Problems of Potential Theory and Their Applications in Engineering, Kluwer, 1991.

[17] R.M. Franks, C.F. Stevens, T.J. Sejnowski, (2003) Independent sources of quantal variability at single glutameric synapses. J. Neur.23(8), pp. 3186-3195.

[18] Gebhardt, C. and Cull-Candy, S.G. (2006) Influence of agonist concentration on AMPA and kainate channels in CA1 pyramidal cells in rat hippocampal slices.J. Physiol. 573, pp. 371394 .

[19] Gerrow K, El-Husseini A.,(2007)Receptors look outward: revealing signals that bring excitation to synapses. Sci STKE. (408):pp56-57

[20] V. Gallo and R. Chittajallu, "Unwrapping Glial Cells from the Synapse: What Lies Inside?", Science 292 (5518), pp.872-873 (2001).

[21] B.Hille, Ionic Channels of Excitable Membranes, 3-rd edtn, Sinauer Associates Inc. Sunderland, MA 2001.

[22] K. Hamdache, M. Labadie (2008) On a reaction-diffusion model for calcium dynamics in dendritic spines. Nonlinear Analysis: Real World Applications. Article in press, already published online doi:10.1016/j.nonrwa.2008.05.005

[23] D. Holcman, Z.Schuss, E. Korkotian (2004) Calcium dynamics in dendritic spines and spine motility. Biophysical Journal Vol. 87, p. 81-91.

[24] D. Holcman, Z. Schuss (2005) Modeling calcium dynamics in dendritic spines. SIAM J. Appl. Math. Vol. 65 No. 3, pp. 1006-1026.

[25] Holcman D, Triller A (2006) Modeling synaptic dynamics driven by receptor lateral diffusion. Biophys J;91:2405-15.

[26] D. Holcman and Z. Schuss, "Escape through a small opening: receptor trafficking in a synaptic membrane", J. of Statistical Physics 117 (5/6), pp.191-230 (2004).

[27] D. Holcman and Z. Schuss, "Diffusion through a cluster of small windows and flux regulation in microdomains", Phys. Lett. A 372, pp.3768-3772 (2008).

[28] D. Holcman and Z. Schuss, "Diffusion escape through a cluster of small absorbing windows", J. Phys. A: Math. and Theoretical 41155001 (2008).

[29] Holmes, W. R. (1995) Modeling the effect of glutamate diffusion and uptake on NMDA and non-NMDA receptor saturation.Biophys. J. 69(5), pp. 1734-47.

[30] J.D. Jackson, Classical Electrodynamics, 3-rd Edition, Wiley, NY (1998).

[31] P. Jonas, G. Major and B. Sakmann, (1993) Quantal components of unitary EPSCs at the mossy fibre synapse on CA3 pyramidal cells of a rat hippocampus J.Physiol. 472, pp. 615-663

[32] E. Kandel, J. Schwartz T. Jessell. Principles of Neural Science (fourth ed.) 2004.

[33] O.D. Kellog, Foundations of Potential Theory, Dover Publications, NY 1954.

[34] Kennedy MB. Signal-processing machines at the postsynaptic density. Science. 2000;290(5492):750-4

[35] T. Kolokolnikov, M. Titcombe and M.J. Ward, "Optimizing the Fundamental Neumann Eigenvalue for the Laplacian in a Domain with Small Traps", European J. Appl. Math. 16, pp.161-200 (2005).

[36] E. Korkotian, D. Holcman, M. Segal, "Dynamic Regulation of Spine-Dendrite Coupling in Cultured Hippocampal Neurons", Euro J. of Neuroscience, 20 (10), pp.2649-63 (2004).

[37] Kusumi A, Y Sako 1996.Cell surface organization by the membrane skeleton. Curr Opin Cell Biol (4):566-74.

[38] Milstein, A. D. Zhou, W. Karimzadegan, S. Bredt, D. S. and Nicoll, R. A. (2007) TARP subtypes differentially and dose-dependently control synaptic AMPA receptor gating.Neuron. 55(6), pp. 905-918.

[39] Madison DV, Malenka RC, Nicoll RA. Mechanisms underlying long-term potentiation of synaptic transmission. Annu Rev Neurosci. 1991;14:379-97.

[40] Malinow, R. AMPA receptor trafficking and long-term potentiation.(2003)Phil. Trans. R. Soc. Lond. B. Biol. Sci.358, pp. 707-714. 
[41] Mattout A, Dechat T, Adam SA, Goldman RD, Gruenbaum Y. Nuclear lamins, diseases and aging. Curr Opin Cell Biol. 2006 Jun;18(3):335-41. 2006

[42] Neher E, Sakmann B, Steinbach JH,The extracellular patch clamp: a method for resolving currents through individual open channels in biological membranes. Pflugers Arch. 1978 Jul $18 ; 375(2): 219-28$.

[43] Nicoll, R A Tomita, S and Bredt, DS (2006) Auxiliary subunits assist AMPA-type glutamate receptors. Science. 311, pp. 1253-1256.

[44] Okamoto, K Nagai, T Miyawaki, A and Hayashi, Y (2004) Rapid and persistent modulation of actin dynamics regulates postsynaptic reorganization underlying bidirectional plasticity. Nat Neurosci. 7(10), pp. 1104-12.

[45] B.L. Patton, A.Y. Chiu, J.R. Sanes, "Synaptic laminin prevents glial entry into the synaptic cleft", Nature 393, pp.698-701 (1998).

[46] I.Yu. Popov, "Extension theory and localization of resonances for domains of trap type", Math. USSR Sbornik 71 (1), pp.209-234 (1992).

[47] Pugh EN Jr, Lamb TD. Amplification and kinetics of the activation steps in phototransduction. Biochim Biophys Acta. 1993 Mar 1;1141(2-3):111-49.

[48] Raghavachari, S. and Lisman, J. E. (2004) Properties of quantal transmission at CA1 synapses.J. Neurophysiol.92(4), pp. 2456-67.

[49] J. W. S. Baron Rayleigh, The Theory of Sound, Vol. 2, 2nd Ed., Dover, New York, 1945.

[50] J. Reingruber D. Holcman, Estimating the rate constant of cyclic GMP hydrolysis by activated phosphodiesterase in photoreceptors, to appear in Journ. Chem. Phys. 2008.

[51] Rosenmund, C.Stern-Bach, Y. Stevens, C. F. (1998) The tetrameric structure of a glutamate receptor channel. Science. 280(5369), pp. 1596-9.

[52] A. Silbergleit, I. Mandel, I. Nemenman, "Potential and field singularity at a surface point charge", J. Math. Phys. 44 (10), pp.4460-4466 (2003).

[53] L.P. Savtchenko and D.A. Rusakov, "The optimal height of the synaptic cleft", Proc. Nat. Acad. Sci. 104 (6), pp.1823-1828 (2007).

[54] P. G. Saffman and M. Delbrück (1975), Brownian motion in biological membranes. Proc. Nat. Acad. Sci. USA 72, 3111-3113.

[55] Sheng, M. Hoogenraad, CC. (2007) The postsynaptic architecture of excitatory synapses: a more quantitative view. Annu Rev Biochem. 76, pp. 823-847.

[56] A. Singer Z, Schuss, D. Holcman, B. Eisenberg, "Narrow Escape I", J. Stat. Phys. 122 (3), pp.437-463 (2006).

[57] A. Singer Z, Schuss, D. Holcman, "Narrow Escape II", J. Stat. Phys. 122 (3), pp.465-489 (2006)

[58] A. Singer Z, Schuss, D. Holcman, "Narrow Escape III", 122 (3), pp.491-509, (2006).

[59] A. Singer and Z. Schuss, "Activation through a narrow opening", Phys. Rev. E (Rapid Comm.) 74, pp.020103(R) (2006).

[60] Singer, A. Schuss, Z. and Holcman, D., Narrow escape and leakage of Brownian particles (to appear in PRE) 2008.

[61] A. Singer, Z. Schuss, A. Osipov and D. Holcman, (2008) Parially reflected diffusion.Siam. J. Appl. Math 68(3), pp. 844-868.

[62] Silver RA, Lubke J, Sakmann B, Feldmeyer D. High-probability uniquantal transmission at excitatory synapses in barrel cortex. Science. 2003;302(5652):1981-4.

[63] Schuss, Z. Singer, A. and Holcman, D. (2007) The narrow escape problem for diffusion in cellular microdomains. Proc. Natl. Acad. Sci. U S A. 104(41), pp.16098-103.

[64] Z. Schuss, Theory and Applications of Stochastic Differential Equations, Wiley Series in Probability and Statistics, Wiley, NY 1980.

[65] Smith, T. C. and Howe, J. R. (2000) Concentration-dependent substate behavior of native AMPA receptors. Nat. Neur. 3(10), pp. 992-7.

[66] Taflia, A Holcman D. The Optimal Post-Synaptic Density for Neurotransmission (submitted).

[67] Taddei A, Hediger F, Neumann FR, Gasser SM. The function of nuclear architecture: a genetic approach. Annu Rev Genet. 2004;38:305-45.

[68] Triller A, D. Choquet Surface trafficking of receptors between synaptic and extrasynaptic membranes: and yet they do move! (2005) Trends Neurosci 28:133-9.

[69] Tsien RY. Fluorescent probes of cell signaling. Annu Rev Neurosci. 1989;12:227-53.

[70] M.J. Ward and J.B. Keller, "Strong Localized Perturbations of Eigenvalue Problems", SIAM J. Appl. Math. 53, pp.770-798 (1993). 
[71] M.J. Ward, W.D. Henshaw and J.B. Keller, Summing Logarithmic Expansions for Singularly Perturbed Eigenvalue Problems, SIAM J. Appl. Math., 53, pp.799-828 (1993).

[72] M.J. Ward and E. Van De Velde,The Onset of Thermal Runaway in Partially Insulated or Cooled Reactors, IMA J. Appl. Math., 48, pp.53-85 (1992).

[73] Xie, X. Liaw, J. Baudry, M.and Berger, T.(1997) Novel expression mechanism for synaptic potentiation: alignment of presynaptic release site and postsynaptic receptor. Proc Natl Acad Sci U S A. 94(13), pp. 6983-8.

[74] R. Zwanzig, (1990) Diffusion-controlled ligand binding to spheres covered by receptors: An effective medium treatment.Proc Natl Acad Sci U S A. 87(15), pp. 5856-7. 
Department of Computational Biology, Ecole Normale Supérieure, 46 rue d'Ulm 75005 PARIS, FranCE. 